\title{
Diagnosis and therapy are walking together on radiopeptides' avenue
}

\author{
Luigi Mansi • Irene Virgolini
}

Published online: 2 March 2011

(C) Springer-Verlag 2011

The paper in this issue by Jiang et al. [1] stimulates a reflection on the state of the art and perspectives in radionuclide therapy, centred on the keyword "peptide". The authors report their preliminary experience on the production of cystine knot peptides (knottins) 2.5D and $2.5 \mathrm{~F}$, engineered to bind with high affinity and specificity to integrin receptors, overexpressed on the surface of a variety of malignant human tumour cells and tumour neovasculature. In this study, $2.5 \mathrm{D}$ and $2.5 \mathrm{~F}$ were successfully labelled with ${ }^{177} \mathrm{Lu}$, representing potential therapeutic agents in a murine model of human glioma xenografts.

Since knottins, small disulphide-rich peptides characterized by a special disulphide through disulphide knot, one of the newest and not yet clinically used molecular forms of peptides, and neoangiogenesis are new areas of clinical interest in humans, we will enlarge our reflection in a wider field; it means that we will also report and analyse older information concerning peptides as tools for diagnosis and therapy, already effective in the present.

The major clinical interest today is devoted to neuropeptides, i.e. to peptides mainly used for diagnosis and therapy of neuroendocrine tumours (NETs). Therefore, we shall focus our attention in this area, structuring as the main

$\overline{\text { Peptide's rap You are a receptor, I am a peptide, I want to interact }}$ with you. I want to be labelled, smoothly radiolabelled to find you. I want to be labelled, roughly radiolabelled to kill you. You are a receptor, I am a peptide, I love and hate you.

L. Mansi $(\bowtie)$

Nuclear Medicine Department, Second University of Naples, P.zza Miraglia 2,

80138 Naples, Italy

e-mail: luigi.mansi@unina2.it

I. Virgolini

Nuclear Medicine Department, Innsbruck Medical University, Innsbruck, Austria

e-mail: irene.virgolini@i-med.ac.at pilaster of our paper directed towards the somatostatin (SST) receptor model. Our contribution will start from the definition of peptide and then briefly describe some of the most interesting applications of this molecular category. We will then consider not only individual perspectives of diagnosis and therapy taken separately, but also how they can be connected between themselves. The reference to the paper of Jiang et al. [1] will be utilized to introduce other intriguing points into the discussion, such as the role of peptides other than those connected to the SST receptor model, with main reference to those of interest to the neoangiogenesis environment, and the relevance of different radioisotopes in the effectiveness of radionuclide therapy.

\section{Definition of peptide}

Peptides (from the Greek $\pi \varepsilon \pi \tau i \delta\llcorner\alpha$, "small digestibles") are short polymers formed from the linking, in a defined order, of two or more $\alpha$-amino acids. The link between one amino acid residue and the next is called an amide bond or a peptide bond. A protein, sometimes referred to as a polypeptide, is a long chain of amino acids joined together in this way. Some proteins contain more than one polypeptide chain. Different interpretations are reported on the number of amino acids that distinguish a peptide from a protein, with the definition of peptide being generally accepted already for molecules constituted by only two amino acids linked with an amide bond.

Using "peptides" as the keyword in PubMed, we found $1,784,203$ and 1,856,490 articles, respectively, in a search performed on 16 June 2010 and repeated on 20 December 2010. Searching separately for peptides and diagnostic imaging, peptides and nuclear medicine, peptides and therapy, peptides and radionuclide therapy, while a relevant percentage of publications on diagnostic imaging 
is connected with nuclear medicine, relatively few are associated with radionuclide applications with respect to the whole number of therapeutic procedures, involving more widely non-radiolabelled peptides. This is a further stimulus to the discussion, defining the possible value of diagnostic imaging as an instrument to predict therapeutic response to peptides both radiolabelled or not.

\section{Why peptides?}

The clinical interest of peptides depends on the presence of a biofunctional activity although a relatively low molecular weight, determining a high extraction and a fast blood clearance after in vivo intravenous administration. Because the first-pass removal is high, a further increase in lesion to background ratio can be achieved after intra-arterial injection. Similarly, a very high tumour to background ratio can be obtained with intra-tumour administration as demonstrated by Heute et al. [2] in a few patients with recurrent brain tumours, treated via a subcutaneous reservoir system that was implanted into the resection cavity.

Moreover, the possible expression on tumour cell membranes of peptide receptors is very wide and homogeneous allowing a favourable distribution with respect to normal cells. As a consequence, an effective in vivo diagnosis and/or therapy with a lower toxicity with respect to traditional strategies can be obtained. Furthermore, the possibility to label the same molecules with different radionuclides (gamma, positron or beta emitters) creates a primary system for a strong connection between diagnosis and therapy, allowing the recruitment for therapeutic purposes only of patients with a high a priori probability to be effectively cured. The diagnostic evidence of an intense pathological uptake can also be used to recruit patients undergoing therapy with nonradiolabelled "cold" peptides.

\section{Which peptide? Which chemical form? Which tracer?}

Because of the presence of thousands of molecules of clinical value in the category "peptides", the first important point to be emphasized is how to optimize the fast transfer from basic research to clinical practice, significantly reducing the number of peptides to be evaluated in clinical trials. An effective answer to this request can be found through the in vivo screening tests of targeted molecular imaging agents. For example, Gagnon et al. [3] demonstrated the feasibility of a high-throughput in vivo screening approach using animal imaging for ${ }^{18} \mathrm{~F}$ peptides.

We then have to remember that the clinical interest in peptides has been growing for many years also outside the oncological field, such as for infection detection [4] or for amyloid cerebral imaging to allow an earlier diagnosis of Alzheimer's disease [5]. Another developing field is related to the research project of de-structuring the antibody $(\mathrm{Ab})$ protein molecule to a "peptide" dimension. It is well known that the whole $\mathrm{Ab}$ is characterized by a high molecular weight determining a low and slow blood clearance not easily allowing an effective diagnosis and therapy. By the way, the "functional" part of the molecule is restricted to the little area that has to interact with the specific antigen. Despite the persistent and sometimes growing clinical interest in Abs there are many proposals and technical solutions trying to reach better results, i.e. a more favourable tumour to background ratio, maintaining the functional capability in littler molecules, i.e. in the "peptide's dimension" [6].

A further possibility to improve the peptide's power to obtain therapeutic results is being pursued, mainly for a more productive action on cerebral lesions, through nanoparticles and/or in the general field of nanotechnologies. This capability can be further increased utilizing the effect on the blood-brain barrier permeability of contrast media for MRI and is therefore particularly interesting as a strategy for cerebral neoplasm [7].

As is well known the concept of "tracer", identifying an in vivo diagnostic probe tracing a biomolecule and allowing its detection from outside of the living body, is not a univocal prerogative of nuclear medicine.

With respect to peptides, great interest is mainly growing, other than in nuclear medicine's kingdom, in optical imaging (OI) and MRI. Concerning OI, i.e. methods based on fluorescence, the major advantage is the highest sensitivity reachable with respect to all the other tracing procedures, allowing the most advanced results achievable in molecular imaging. Being based on the light as signal, the major disadvantage is related to the capability to perform only an analysis of surfaces and not in depth. Nevertheless, it has been reported how its clinical use can be strongly supported in areas of endoscopy or in the field of guided surgery. Using OI, the capability to reach a very high accuracy in defining the full resectability has been demonstrated for a tumour during the surgical procedure [8]. An improvement in intra-surgical staging at the level of areas such as pleura, peritoneum and, probably, lymph nodes could be also hypothesized. To increase the potential of clinical applications, bifunctionally chelated peptides allowing both fluorescence and positron emission tomography (PET) have been produced and already used in animal imaging [9]

\section{The somatostatin receptor system in the diagnostic and therapeutic scenario}

The use of somatostatin receptor (SSTR) imaging and therapy (peptide receptor radionuclide therapy, PRRT) in 
routine clinical practice is scientifically based on the enhanced expression of SSTR on tumour cells as compared to the normal ones [10]. As neuropeptides, SST analogues link to specific receptors on the cell membrane, becoming diagnostic probes when radiolabelled. Moreover, they can have a therapeutic action per se on hormonal hypersecretion and/or on tumour growth. When internalized, they can exert a stronger therapeutic efficacy, acting as vectors of chemotherapeutics or of radioactivity, the latter also determining a wider effect on adjacent cells within the radiation range and/or through an action on the surrounding stroma cells.

The major value of the SST system derives from the "chemical evolution" of the "native molecule", affected by a overly fast blood clearance not allowing a therapeutic efficacy. The pivotal role of the octreotide analogue and of ${ }^{111}$ In-DTPA-D-Phe ${ }^{1}$-pentetreotide (OctreoScan ${ }^{\circledR}$, OCT) has been further increased by the availability of other peptides labelled with gamma emitters, together with a large series of radiocompounds labelled with positron or beta emitters, allowing PET studies as well as therapy (for review see [[10]]). The great methodological advantage is derived from the possibility to use almost the same molecular structure both for diagnostic and therapeutic purposes, creating conditions for the "ideal model" in radionuclide therapy, where the recruitment of the patient that will benefit from therapy can be predicted a priori through a diagnostic whole-body scan. Therefore, as for radioiodine in differentiated thyroid cancer, diagnosis and therapy can walk together also on the peptides' avenue.

At present, there is research interest in finding the best SST analogue having the capability to link to all five isolated and characterized SSTR. In this way, it could be possible to increase the diagnostic sensitivity also in NETs not expressing $\mathrm{SSTR}_{2}$ and $\mathrm{SSTR}_{5}$ (which are the main targets for octreotide), such as in insulinoma. As an alternative, a wider sensitivity can be achieved using other neuropeptides such as bombesin, vasoactive intestinal polypeptide (VIP), pituitary adenylate cyclase-activating polypeptide (PACAP), cholecystokinin (CCK), gastrinreleasing peptide (GRP), gastrin, neurotensin, neuropeptide $\mathrm{Y}$ (NPY), substance $\mathrm{P}$, oxytocin, luteinizing hormonereleasing hormone (LH-RH), glucagon-like peptide 1 (GLP-1), calcitonin, endothelin, atrial natriuretic factor and alpha-melanocyte-stimulating hormone $(\alpha-\mathrm{MSH})$ [11].

In clinical diagnostic imaging the best results, at the present, can be achieved using SST analogues labelled with ${ }^{68} \mathrm{Ga}$, through a DOTA chelation $[12,13]$. Slight differences in kinetics and sensitivity have been observed changing both the chelate or the peptide's molecule, also including lanreotide [14]. New proposals, with a possible clinical interest, are also connected with ${ }^{99 \mathrm{~m}} \mathrm{Tc}$-labelled radiocompounds, using gastrin peptide-hydrazinonicotinamide (HYNIC) as chelator [15].
The research on alternative radionuclides is following the interesting perspective based on the use of peptides labelled with ${ }^{64} \mathrm{Cu}[16]$, allowing both PET and therapy. Less promising are peptides labelled with radioiodine or ${ }^{18} \mathrm{~F}$, despite the easy radiolabelling using halogens. The reason at first depends on an in vivo de-iodination or defluorination creating sometimes unreliability in defining a satisfactory tumour to background ratio. But the biggest problem is "the lack of the advantage" referred to the substantial identity in the molecular structure and pharmacokinetics between diagnostic and therapeutic tools, as it happens for DOTA peptides. In other words, radioiodinated and radiofluorinated diagnostic tracers cannot walk together with therapeutic ones. As a further diagnostic radionuclide of clinical interest, the positron emitter ${ }^{86} \mathrm{Y}$ has to be remembered. Despite its isotope identity with ${ }^{90} \mathrm{Y}$, one of the most widely used therapeutic isotopes, this radionuclide did not find widespread application because of economic problems in its production. This is one of the reasons determining a possible survival of ${ }^{111}$ In-pentetreotide, although affected by a lower diagnostic accuracy as opposed to PET radiopeptides. ${ }^{111}$ In-labelled SST analogues could be considered an advantageous alternative to PET both in oncology or better when spatial resolution is not a major request, as in the definition of activity in benign diseases such as Graves' exophthalmus, rheumatoid arthritis or sarcoidosis, having also therapeutic implications [17]. Similarly, an effective role can be maintained in patients with pituitary adenomas, to justify an expensive and not always effective therapy with SST analogues. Furthermore, OCT also has a reliable role for dosimetry, derived from the longer half-life of ${ }^{111} \mathrm{In}$ versus ${ }^{68} \mathrm{Ga}$. Indications only available with the ${ }^{111}$ In-labelled SST analogue, but not with ${ }^{68} \mathrm{Ga}$-labelled, have also been proposed for radioguided surgery, independent of the tumour-specific receptor uptake. For example, interesting results can be obtained in surgical staging of operable lung tumours, both in carcinoids and non-small cell carcinoma, where the intense uptake is not dependent on the tumour per se, but on the peri-tumour reactive inflammatory cells [17].

\section{Alternatives to somatostatin analogues}

As previously reported, in the class of neuropeptides there are many molecules other than SST analogues that can be used for diagnostic and therapeutic purpose in NETs. However, none of these reached an accuracy able to substitute the SST analogues. In fact, because of the favourable results obtained by OCT and of the major improvement allowed by PET with ${ }^{68} \mathrm{Ga}$-DOTA peptides, there has been no significant clinical interest in those, apart from some peculiar situations such as in the diagnosis of insulinoma and in staging/restaging of 
thyroid medullary carcinoma (TMC). Similarly, the availability of ${ }^{99 \mathrm{~m}}$ Tc-labelled peptides, despite the well-known advantages of this radionuclide, did not obtain dominance not only with regard to ${ }^{68} \mathrm{Ga}$, but also in comparison with

${ }^{111}$ In-pentetreotide, because of the lack of a significant diagnostic improvement.

With respect to TMC, where SST analogues show a low sensitivity in detecting all lesions, although promising results were obtained using other radiocompounds, none of them has been individuated as an effective tracer to be used alone in these patients. The reason is probably due both to the polyclonal character of the malignant cells in TMC and to the escape phenomenon, creating a nonhomogeneous receptor's distribution on malignant lesions. Therefore, because of the presence of many different targets, there are no effective bullets able to reach and destroy alone all malignant cells.

Although it is not for peptide, major interest is directed toward ${ }^{18} \mathrm{~F}$-dihydroxyphenylalanine (F-dopa), an amino acid precursor of dopamine [18]. Neuroendocrine tumours (APUD) are characterized by an increased amine precursor uptake; therefore, F-dopa shows similar, although not completely identical, kinetics as compared to metaiodobenzylguanidine (MIBG), which can be a therapeutic agent when labelled with ${ }^{131}$ I. Using F-dopa, preliminary results showed an increased sensitivity in NETs compared to MIBG and OctreoScan ${ }^{\circledR}$. Similarly to DOTA peptides, Fdopa presents a higher sensitivity for differentiated NET lesions, although obtained through the different uptake mechanism which may result in mismatches. Being at present more easily available and cheaper, DOTA peptides can be considered the first choice in NETs, except for tumours not presenting a favourable SSTR expression, such as insulinoma. A wider experimental demonstration of a similar behaviour between F-dopa and ${ }^{131}$ I-MIBG could create an interesting perspective for the utilization of $\mathrm{F}$ dopa as a priori tracer for the therapeutic efficacy in patients with NETs. This perspective, connecting diagnostic F-dopa and therapeutic ${ }^{131}$ I-MIBG walking together, could add a further effective bullet in the fight against NETs not treatable by SST analogues.

As final perspectives concerning a diagnostic scenario walking together with therapy we have to briefly report two other fields of research that are ready to enter into clinical practice: (1) radiolabelling of peptides with ${ }^{64} \mathrm{Cu}$ and (2) radiopeptides to visualize (and treat) neoangiogenesis.

With regard to the first ones, great interest is related to the physical characteristics of ${ }^{64} \mathrm{Cu}$, a positron emitter also emitting beta radiation. Therefore, using such radiocompounds, both a PET and (at higher doses) a radionuclide therapy could be achieved.

With regard to the imaging of neoangiogenesis many peptides, such as ${ }^{18} \mathrm{~F}$-Galacto-RGD, are almost ready for clinical utilization $[19,20]$. The great interest in these tracers is derived from the relevant importance of neovasculature in tumour growth. Therefore, uptake is not only a good diagnostic marker of malignancy, but also defines a very interesting target for therapeutic bullets. The main advantage is related to the capability to produce a local radiotherapy determining tumour destruction, because of an almost specific action on the vascular neoplastic stroma. It means that also cellular components not expressing a receptor target can be destroyed, if a major reduction in blood flow is determined. Therefore, patients showing RGD uptake can be recruited for a therapeutic approach using molecular therapeutic agents. Moreover, increasing the number of neovessels together with malignancy, this therapy could act as a complementary tool as opposed to SST analogues, the latter having effect mainly on differentiated lesions. In this way, an integrated and more effective therapeutic strategy could be allowed. In fact, the probability and the intensity of RGD uptake tend to increase with malignancy, because neoangiogenesis is related mainly to a fast growing and/or undifferentiated neoplasm.

Since our interest in this paper is to discuss our issues in a clinical scenario, before proceeding to conclusions we want to briefly report the state of the art of radionuclide therapy in NETs, to find where and how it could be possible to improve actual strategies.

\section{Radionuclide therapy of NETs (PRRT)}

Patients are subjected to PRRT on the basis of diagnostic evidence of in vivo uptake allowing an a priori recruitment only of individual patients who will benefit from therapy.

\section{Intravenous treatment}

In general, since its first implementation by the Rotterdam group in 1994 [21], only a few peer-reviewed papers have been published on PRRT in a larger cohort of patients over the last 15 years. On the other hand, and to be more critical, there exist abundant numbers of abstracts, textbook articles as well as review articles on PRRT by various groups, worldwide, that have been published over the last years. In the following we will stick to recent publications in the peer-reviewed journals only.

Today, ${ }^{90} \mathrm{Y}$ and ${ }^{177} \mathrm{Lu}$ are the two radionuclides used for PRRT with SST analogues. ${ }^{111}$ In-DTPA-D-Phe ${ }^{1}$-pentetreotide $\left(\right.$ OctreoScan $\left.{ }^{\circledR}\right)$ was first pioneered for PRRT, but was subsequently displaced by radiolabelled DOTATOC and DOTATATE derivates. This is due to the fact that ${ }^{111} \mathrm{In}$ labelled peptides are not ideal for PRRT because of the small particle range, and thus a short tissue penetration. 
Unfortunately, and sad to be said, having past the 15year anniversary of PRRT, for none of the current peptides used for PRRT is a randomized clinical trial yet available. The treatment cycle dose, cycle interval, cumulative dose, etc. are based on only a few studies reported in larger cohorts of patients. Mostly, the treatment schemes depend on local expertise and clinical judgement. Treatment with radiolabelled SST analogues is usually discontinued if the tumour progresses. Furthermore, WHO grade 3 or 4 side effects are a reason to reduce the cycle dose, or even to discontinue PRRT. In general, such side effects are rare.

Long-term expertise seems to remain limited to the larger well-known PRRT centres in Europe (namely the centres in Rotterdam, Basel, Milan, Bad Berka, Innsbruck/ Vienna) and in Iowa. The ENETS Consensus Guidelines for the Standards of Care in NETs [22] tried to define the minimum requirements for patients eligible for PRRT, based on the fact that at the different centres different SST analogues are being used, and their availability depends on national law and local permissions. Among these criteria, high uptake by the tumour lesions, inoperable disease, a Karnofsky score $>50 \%$ and a life expectancy of at least 3-6 months are required. Contraindications are an impaired renal function (i.e. a creatinine clearance of $<40-50 \mathrm{ml} / \mathrm{min}$ ), an impaired haematological function $(\mathrm{Hgb}<8 \mathrm{~g} / \mathrm{dl}$; platelets $<75 \times 10 \mathrm{E} 9 / 1 ;$ WBC $<2 \times 10 \mathrm{E} 9 / 1)$, and severe hepatic and cardiac impairment.

More recently reported studies for ${ }^{90}$ Y-DOTATOC showed a significant therapeutic benefit for PRRT, confirming former reports from the Basel, Milan, Rotterdam, Innsbruck, Bad Berka and Iowa groups [10, 23] with an overall response rate of about $80 \%$.

In patients relapsing after PRRT with ${ }^{90} \mathrm{Y}$-DOTATOC the ${ }^{177}$ Lu-labelled DOTATOC may be an alternative further treatment option that also was proven to be clinically safe and efficacious [24]. Efficacy of PRRT with consecutive radiolabelled SST analogues such as ${ }^{90}$ Y-DOTA-lanreotide followed by ${ }^{90}$ Y-DOTATOC [30], or ${ }^{90}$ Y-DOTATOC followed by ${ }^{177} \mathrm{Lu}$-DOTATATE in sequential order, or in combined order was also demonstrated [25, 26].

The octreotate analogues differ from the octreotide analogues only in that the C-terminal threoninol is replaced with threonine, but do have a higher tumour uptake. ${ }^{177} \mathrm{Lu}$ DOTATATE resulted in an $80 \%$ overall response as reported Kwekkeboom et al. [27]. In this study in 310 patients with NETs up to $30 \mathrm{GBq}$ were applied in up to 4 cycles with a 6- to 10 -week interval in between. Median time to progression was 40 months from start of treatment in 249 patients who either had complete response (CR)/ partial response (PR)/minimal response (MR) or stable disease (SD). Median overall survival was 46 months from start of therapy. The authors also compared similar patient subgroups from different interventional and observational studies, and there seemed to be a survival benefit of 3.5-6 years! The Innsbruck group confirmed these results with an overall response rate of $76 \%$ in 55 patients of whom 15 patients had been already pretreated with ${ }^{90}$ Y-DOTATOC [24]. The efficacy of PRRT in 60 patients with progressive gastroenteropancreatic (GEP) NETs with ${ }^{90}$ Y-DOTATATE was also proven by Cwikla et al. [28] who reported PR in $23 \%$ and SD in $77 \%$ after 6 months follow-up.

Locoregional treatment

Locoregional application should provide the application of a higher dose to the tumour lesions. Furthermore, it might convert unresectable primary tumours to resectable ones. However, no randomized PRRT data are yet available for any of the SST analogues applied locally.

In fact, the locoregional application of PRRT should be more effective with a lesser irradiation dose (and thus lower rate of side effects) than seen after the systemic application, for bone marrow and kidneys. Comparative semiquantitative dose estimates were recently demonstrated by the Heidelberg group using ${ }^{68} \mathrm{Ga}$-DOTATOC PET [29]. In this study, the authors demonstrate that SST analogues show a 3.75-fold higher standardized uptake value (SUV) in liver metastases after i.a. infusion into the hepatic artery as compared to i.v. infusion in the same patient. These data are very promising for a more selective alternative to transarterial chemoembolization or selective internal radiation therapy for liver metastases. Recently, Limouris et al. [30] reported that the selective intra-arterial hepatic infusion of ${ }^{111}$ In-DTPA-OCT in inoperable metastasized liver patients (given in up to 12 cycles; $6.3-/-2.3 \mathrm{GBq}$ ) resulted in $47 \%$ $\mathrm{PR}$, in $18 \% \mathrm{SD}$, and one patient even showed a CR. The estimated median survival time was about 3 years in this report, which is fairly high. A $79 \%$ overall response rate to intra-hepatic ${ }^{90}$ Y-DOTA-lanreotide was found also by McStay et al. [31]. Both the compounds do not seem optimal for locoregional PRRT, and the ${ }^{90} \mathrm{Y}$ - or ${ }^{177} \mathrm{Lu}$ labelled analogues may provide better results. With regard to intra-arterially given ${ }^{90} \mathrm{Y}$-labelled microspheres which are nowadays also applied for treatment of liver metastases in NET patients [32], a randomized study of selective internal radiation therapy (SIRT) versus or plus/minus PRRT is warranted.

Not only NET patients were subjected to locoregional therapy. In a recent short communication Heute et al. [2] showed a clear response to locoregional application of ${ }^{90} \mathrm{Y}$ DOTATOC in a few patients with recurrent brain tumours that were treated via a subcutaneous reservoir system (Rickham reservoir) that was implanted into the resection cavity. Because PRRT can safely be repeated several times, the total dose to the tumour tissue surpasses the dose of a single standard whole-brain radiotherapy session by a factor 
of 10 or more. Furthermore, locoregional PRRT may be superior to local chemotherapy which is limited to penetration of the tumour whereas PRRT using B-particles reach even $5-10 \mathrm{~mm}$ beyond the point of penetration.

\section{Combination treatments}

Long-acting octreotide (30 mg intramuscular every 4 weeks) has recently been shown to inhibit tumour progression in patients with metastasized well-differentiated midgut NETs [33] and may therefore have a potential role in the concomitant treatment of these patients. In fact, the Innsbruck protocol $[24,34]$ uses two intramuscular injections of long-acting SST analogues, 4 weeks apart from each other, between application of two PRRT cycles.

Because of the differences in the biodistribution and critical organs, targeted therapy such as combined therapy of radiopharmaceuticals could provide significant increase in the delivered tumour dose over either agent alone. The magnitude of increase depends on the relative dose delivered by each agent. PRRT might be improved by a combination of ${ }^{177} \mathrm{Lu}$ - and ${ }^{90}$ Y-labelled SST radiopharmaceuticals [32]. Furthermore, a potential advantage of combined ${ }^{131}$ I-MIBG and ${ }^{90}$ Y-DOTATOC therapy was proposed by the Iowa group [35] suggesting that the optimal tumour dose for the combined agents may be achieved when the dose per activity delivered to the tumour by ${ }^{90}$ Y-DOTATOC is $2-3$ times that of ${ }^{131} \mathrm{I}$-MIBG.

With the increasing advancements in genomic and proteomic research our understanding of the basic mechanisms underlying the development and progression of the various NET entities will improve. Further developments may include new multimodal concepts, maybe leading to pathway-specific therapeutic strategies tailored to the individual biological background of the patient. Targeted therapy combinations have demonstrated unique promises and also provide challenges related to timing, dosage necessary to enhance efficacy and reduce overlapping toxicities. There is emerging interest in the oncological community and industry in combining therapeutic radiopharmaceuticals with other modalities such as immunotherapy, radiotherapy or chemotherapy. Incorporation of PRRT into clinical protocols in combination with chemotherapy, immunotherapy or external beam radiation is an evolving new treatment option of future targeted therapy protocols. Many other combination possibilities are evolving and finding their way towards clinical trials.

Among many possibilities, the expression of tyrosine kinase receptors on NETs provides the basis for new tyrosine kinase inhibitors such as sunitinib concomitantly used in future treatment protocols. Other molecules include angiogenesis inhibitors (such as bevacizumab) which may alone, or in combination with cytotoxic agents, have some significance. Rapamycin (everolimus), an oral inhibitor of the mammalian target of rapamycin (mTOR) signalling pathway alone, or in combination with cytotoxic treatment, might become of value for treatment in NETs [36].

\section{Conclusions}

According to the results obtained so far, there can be no doubt about the wide therapeutic index and the high efficacy of SST analogues in the symptomatic management of NETs. In addition, the results of PRRT with long-acting SST analogues indicate that these molecular therapies have their place in the treatment of patients with SST receptorpositive NETs for size reduction, improvement of quality of life and overall prognosis. Results of PRRT are encouraging in a few of the limited number of alternative treatment approaches. Serious side effects are rare, especially in combination with amino acids for kidney protection. Patients should always be evaluated by preceding SST scintigraphy and dosimetry using respective octreotide or lanreotide analogues, preferably the ${ }^{68} \mathrm{Ga}$-labelled ones for PET. ${ }^{18}$ F-fluorodeoxyglucose (FDG) PET scanning shows a poor sensitivity to detect NETs with a low metabolic activity and slow growth rate, while together with the ${ }^{68} \mathrm{Ga}$ labelled SST analogues ${ }^{18}$ F-FDG PET has clinical potential for the metabolic restaging of patients undergoing PRRT. The role of other PET radiopharmaceuticals, ${ }^{11} \mathrm{C}$-5-hydroxytryptophan (HTP) and ${ }^{18} \mathrm{~F}$-L-dopa, for patients with NETs has to be comparatively assessed, and tumours may show unbelievable variation.

In general, there is a need for randomized PRRT trials in order to establish which treatment scheme and which radiolabelled SST analogue, or combination of analogues, is optimal for PRRT. A great demand for multi-institutional trials on the concomitant use of molecular therapeutics is also evolving, whereas the availability of adequate facilities and legislation are restricting progress. One of the major problems is still the availability of these radiopeptides in general, and the request for new and cheaper therapeutic radionuclides in particular. For certain tumour entities ${ }^{131} \mathrm{I}$ MIBG therapy is appropriate, and combination therapies with SST analogues are being discussed. Problems include the process of selection of patients (histopathology, disease burden: stable disease versus progressive disease), the assessment of response (CT/MRI/US criteria, sensitivity for detection of response, radionuclide imaging, tumour markers, quality of life), the dose limiting toxicity and finally the cost of trials.

The future treatment of NETs will be more individualized where the tumour biology and molecular genetics play a major role. Future therapeutic trials should discuss the possibility of including patients at an earlier stage of 
disease, and therefore being able to evaluate the possible potential of PRRT at an earlier stage of the tumour disease.

If a more widespread use of PRRT can be guaranteed, such therapy may well become the therapy of first choice in patients with metastasized or inoperable GEP NETs and other tumours expressing SST receptors in high quantity.

Phase III trials are needed to confirm the results and combinations of different treatment modalities are needed to make optimal use of the new modalities. Whether the new therapies prolong survival of the patients should also be established.

The definition of response has changed. Today we consider "stabilization of disease" as a response to PRRT. In fact, this has been applied for a long while with the socalled biotherapy (SST and interferon analogues). But now, response criteria may be changing. A CT/MRI-documented progression - is the tumour really progressive? Not to be too provocative: does a positive ${ }^{18}$ F-FDG PET scan really imply progression in a ${ }^{68} \mathrm{Ga}$-SSTR PET-positive scan?

All future improvements in therapy have to be accompanied by a parallel development of diagnostic procedures. In this direction, together with the diagnostic/therapeutic SST analogue family, companions of travel, such as F-dopa and MIBG, radiocompounds labelled with ${ }^{64} \mathrm{Cu}$ or radiotracers seeking (and having as therapeutic target) neovessels could give significant support in winning the mother of all battles, the war against cancer.

\section{References}

1. Jiang L, Miao Z, Kimura RH, Liu H, Cochran JR, Culter CS, Bao A, Li P, Cheng Z. Preliminary evaluation of (177)Lu-labeled knottin peptides for integrin receptor-targeted radionuclide therapy. Eur J Nucl Med Mol Imaging 2010. doi: 10.1007/s00259-010-1684-x.

2. Heute $\mathrm{D}$, Kostron $\mathrm{H}$, von Guggenberg E, Ingorokva S, Gabriel M, Dobrozemsky G, et al. Response of recurrent high-grade glioma to treatment with (90)Y-DOTATOC. J Nucl Med 2010;51(3):397-400.

3. Gagnon MK, Hausner SH, Marik J, Abbey CK, Marshall JF, Sutcliffe JL. High-throughput in vivo screening of targeted molecular imaging agents. Proc Natl Acad Sci U S A 2009;106 (42):17904-9.

4. Lupetti A, Nibbering PH, Welling MM, Pauwels EK. Radiopharmaceuticals: new antimicrobial agents. Trends Biotechnol 2003;21(2):70-3.

5. Jang H, Arce FT, Ramachandran S, Capone R, Azimova R, Kagan BL, et al. Truncated beta-amyloid peptide channels provide an alternative mechanism for Alzheimer's disease and Down syndrome. Proc Natl Acad Sci U S A 2010;107(14):6538-43.

6. Olafsen $\mathrm{T}, \mathrm{Wu} \mathrm{AM}$. Antibody vectors for imaging. Semin Nucl Med 2010;40(3):167-81.

7. Olson ES, Jiang T, Aguilera TA, Nguyen QT, Ellies LG, Scadeng $\mathrm{M}$, et al. Activatable cell penetrating peptides linked to nanoparticles as dual probes for in vivo fluorescence and MR imaging of proteases. Proc Natl Acad Sci U S A 2010;107(9):4311-6.

8. Segala J, Engelman DM, Reshetnyak YK, Andreev OA. Accurate analysis of tumor margins using a fluorescent $\mathrm{pH}$ low insertion peptide (pHLIP). Int J Mol Sci 2009;10(8):3478-87.
9. Kimura RH, Miao Z, Cheng Z, Gambhir SS, Cochran JR. A duallabeled knottin peptide for PET and near-infrared fluorescence imaging of integrin expression in living subjects. Bioconjug Chem 2010 Feb 4

10. Virgolini IJ, Gabriel M, von Guggenberg E, Putzer D, Kendler D, Decristoforo C. Role of radiopharmaceuticals in the diagnosis and treatment of neuroendocrine tumours. Eur J Cancer 2009;45 Suppl 1:274-91.

11. Mariani G, Erba PA, Signore A. Receptor-mediated tumor targeting with radiolabeled peptides: there is more to it than somatostatin analogs. J Nucl Med 2006;47(12):1904-7.

12. Ambrosini V, Tomassetti P, Franchi R, Fanti S. Imaging of NETs with PET radiopharmaceuticals. Q J Nucl Med Mol Imaging 2010;54(1):16-23.

13. Gabriel M, Decristoforo C, Kendler D, Dobrozemsky G, Heute D, Uprimny C, et al. 68Ga-DOTA-Tyr3-octreotide PET in neuroendocrine tumors: comparison with somatostatin receptor scintigraphy and CT. J Nucl Med 2007;48(4):508-18.

14. Traub-Weidinger T, Von Guggenberg E, Dobrozemsky G, Kendler $\mathrm{D}$, Eisterer W, Bale R, et al. Preliminary experience with (68)GaDOTA-lanreotide positron emission tomography. Q J Nucl Med Mol Imaging 2010;54(1):52-60.

15. King R, Surfraz MB, Finucane C, Biagini SC, Blower PJ, Mather SJ. 99mTc-HYNIC-gastrin peptides: assisted coordination of $99 \mathrm{mTc}$ by amino acid side chains results in improved performance both in vitro and in vivo. J Nucl Med 2009;50(4):591-8.

16. Boswell CA, Regino CA, Baidoo KE, Wong KJ, Milenic DE, Kelley JA, et al. A novel side-bridged hybrid phosphonate/acetate pendant cyclam: synthesis, characterization, and $64 \mathrm{Cu}$ small animal PET imaging. Bioorg Med Chem 2009;17(2):548-52.

17. Cascini GL, Cuccurullo V, Mansi L. The non tumour uptake of (111)In-octreotide creates new clinical indications in benign diseases, but also in oncology. Q J Nucl Med Mol Imaging 2010;54(1):24-36.

18. Putzer D, Gabriel M, Kendler D, Henninger B, Knoflach M, Kroiss A, et al. Comparison of (68)Ga-DOTA-Tyr(3)-octreotide and (18)F-fluoro-L-dihydroxyphenylalanine positron emission tomography in neuroendocrine tumor patients. Q J Nucl Med Mol Imaging 2010;54(1):68-75.

19. Haubner R, Kuhnast B, Mang C, Weber WA, Kessler H, Wester HJ, et al. [18F]Galacto-RGD: synthesis, radiolabeling, metabolic stability, and radiation dose estimates. Bioconjug Chem 2004;15(1):61-9.

20. Cai W, Chen X. Multimodality molecular imaging of tumor angiogenesis. J Nucl Med 2008;49 Suppl 2:113S-28S.

21. Krenning EP, Kooij PP, Bakker WH, Breeman WA, Postema PT, Kwekkeboom DJ, et al. Radiotherapy with a radiolabeled somatostatin analogue, [111In-DTPA-D-Phe1]-octreotide. A case history. Ann N Y Acad Sci 1994;733:496-506.

22. Kwekkeboom DJ, Krenning EP, Lebtahi R, Komminoth P, KosKudła B, de Herder WW, et al. ENETS Consensus Guidelines for the Standards of Care in Neuroendocrine Tumors: peptide receptor radionuclide therapy with radiolabeled somatostatin analogs. Neuroendocrinology 2009;90(2):220-6.

23. Kwekkeboom DJ, de Herder WW, van Eijck CHJ, Kam BL, van Essen M, Teunissen JJM, et al. Peptide receptor radionuclide therapy in patients with gastroenteropancreatic neuroendocrine tumours. Semin Nucl Med 2010;40:78-88.

24. Gabriel M, Andergassen U, Putzer D, Kroiss A, Waitz D, von Guggenberg E, et al. Individualized peptide-related-radionuclidetherapy concept using different radiolabelled somatostatin analogs in advanced cancer patients. Q J Nucl Med Mol Imaging 2010;54:92-9.

25. Forrer F, Uusijärvi H, Storch D, Maecke HR, Mueller-Brand J. Treatment with $177 \mathrm{Lu}-D O T A T O C$ of patients with relapse of neuroendocrine tumours after treatment with 90Y-DOTATOC. J Nucl Med 2005;46:1310-16. 
26. Seregni E, Maccauro M, Coliva A, Castellani MR, Bajetta E, Aliberti G, et al. Treatment with tandem [(90)Y]DOTA-TATE and [(177)Lu]DOTA-TATE of neuroendocrine tumors refractory to conventional therapy: preliminary results. Q J Nucl Med Mol Imaging 2010;54:84-91.

27. Kwekkeboom DJ, de Herder WW, Kam BL, van Eijck CH, van Essen M, Kooij PP, et al. Treatment with the radiolabeled somatostatin analog [177Lu-DOTA0, Tyr3]octreotate: toxicity, efficacy, and survival. J Clin Oncol 2008;26:2124-30.

28. Cwikla JB, Sankowski A, Seklecka N, Buscombe JR, Nasierowska-Guttmejer A, Jeziorski $\mathrm{KG}$, et al. Efficacy of radionuclide treatment DOTATATE Y-90 in patients with progressive metastatic gastroenteropancreatic neuroendocrine carcinomas (GEP-NETs): a phase II study. Ann Oncol 2010;21 (4):787-94.

29. Kratochwil C, Giesel FL, López-Benítez R, Schimpfky N, Kunze $\mathrm{K}$, Eisenhut $\mathrm{M}$, et al. Intraindividual comparison of selective arterial versus venous $68 \mathrm{Ga}-\mathrm{DOTATOC} \mathrm{PET} / \mathrm{CT}$ in patients with gastroenteropancreatic neuroendocrine tumors. Clin Cancer Res 2010;16(10):2899-905.

30. Limouris GS, Chatziioannou A, Kontogeorgakos D, Mourikis D, Lyra M, Dimitriou P, et al. Selective hepatic arterial infusion of In111-DTPA-Phe1-octreotide in neuroendocrine liver metastases. Eur J Nucl Med Mol Imaging 2008;35:1827-37.

31. McStay MKG, Maudgil D, Williams M, Tibballs JM, Watkinson AF, Caplin ME, et al. Large-volume liver metastases from neuroendo- crine tumors: hepatic intraarterial 90Y-DOTA-lanreotide as effective palliative therapy. Radiology 2005;237:718-26.

32. Kalinowski M, Dressler M, König A, El-Sheik M, Rinke A, Höffken $\mathrm{H}$, et al. Selective internal radiotherapy with yttrium-90 microspheres for hepatic metastatic neuroendocrine tumors: a prospective single center study. Digestion 2009;79(3):137-42.

33. Rinke A, Müller HH, Chade-Brittinger C, Klose KJ, Barth P, Wied $\mathrm{M}$, et al. Placebo-controlled, double-blind, prospective, randomized study on the effect of octreotide LAR in the control of tumor growth in patients with metastatic neuroendocrine midgut tumors: a report from the PROMID Study Group. J Clin Oncol 2009;27(28):4656-63.

34. Gabriel M, Oberauer A, Dobrozemsky G, Decristoforo C, Putzer D, Kendler D, et al. 68Ga-DOTA-Tyr3-octreotide PET for assessing response to somatostatin-receptor-mediated radionuclide therapy. J Nucl Med 2009;50(9):1427-34.

35. Madsen MT, Bushnell DL, Juweid ME, Menda Y, O'Dorisio MS, O'Dorisio T, et al. Potential increased tumor-dose delivery with combined 131I-MIBG and 90Y-DOTATOC treatment in neuroendocrine tumors: a theoretic model. J Nucl Med 2006;47(4):660-7.

36. Kulke MH, Siu LL, Tepper JE, Fisher G, Jaffe D, Haller DG, Ellis LM, Benedetti JK, Bergsland EK, Hobday TJ, Van Cutsem E, Pingpank J, Oberg K, Cohen SJ, Posner MC, Yao JC. Future Directions in the Treatment of Neuroendocrine Tumors: Consensus Report of the National Cancer Institute Neuroendocrine Tumor Clinical Trials Planning Meeting. J Clin Oncol 2011 Jan 24 [Epub ahead of print]. 